\title{
The Lagrangian exploration module: An apparatus for the study of statistically homogeneous and isotropic turbulence
}

\author{
Robert Zimmermann, ${ }^{1, a)}$ Haitao Xu, ${ }^{1, b)}$ Yoann Gasteuil, ${ }^{2}$ Mickaël Bourgoin, ${ }^{3}$ Romain Volk, ${ }^{2}$ \\ Jean-François Pinton, ${ }^{2}$ and Eberhard Bodenschatz ${ }^{1}$ \\ (International Collaboration for Turbulence Research) \\ ${ }^{1}$ Max Planck Institute for Dynamics and Self-Organization, Göttingen D-37077, Germany \\ ${ }^{2}$ Laboratoire de Physique, CNRS and Ecole Normale Supérieure de Lyon, \\ UMR 5672, Lyon F-69007, France \\ ${ }^{3}$ Laboratoire des Ecoulements Geophysiques et Industriels, CNRS UJF INPG, UMR5519, \\ Grenoble F-38041, France
}

(Received 31 December 2009; accepted 21 April 2010; published online 28 May 2010)

\begin{abstract}
We present an apparatus that generates statistically homogeneous and isotropic turbulence with a mean flow that is less than $10 \%$ of the fluctuating velocity in a volume of the size of the integral length scale. The apparatus is shaped as an icosahedron where at each of the 12 vertices the flow is driven by independently controlled propellers. By adjusting the driving of the different propellers the isotropy and homogeneity of the flow can be tuned, while keeping the mean flow weak.
\end{abstract}

(C) 2010 American Institute of Physics. [doi:10.1063/1.3428738]

\section{INTRODUCTION}

Fully developed fluid turbulence is most easily studied under the assumption of statistical homogeneity and isotropy. ${ }^{1}$ This is hard to realize in laboratory flows and basically never found in naturally occurring flows. In experiments it would be desirable to implement a situation, in which the conditions such as the homogeneity or the isotropy of the flow can be tuned. Additionally, such experiments should allow simultaneous Eulerian and Lagrangian measurements. The latter have captured renewed interest in the community $^{2-6}$ as they provide new insights into turbulent mixing and transport. Lagrangian studies require the measurement volume to follow the mean flow, while local measurements, such as hot wire or laser Doppler velocimetry, are sufficient to measure Eulerian statistics under the assumption of Taylor's frozen flow hypothesis.

Traditionally, grid generated turbulence in wind tunnels has been the first choice for experiments. There the statistics of the decaying turbulence have been observed to be close to homogeneous and isotropic. Recently active grids have provided an increased Reynolds number and some control on the flow properties. ${ }^{7,8}$ Although wind tunnel flows are ideally suited for Eulerian measurements with local probes, the typically fast mean flow velocities make Lagrangian measurements difficult. Additionally, in conventional wind tunnels the maximum available observation time, given by the length of the tunnel divided by the mean velocity of the flow, is short. Thus, even if the measurement volume would move with the mean flow, it is rarely possible to follow Lagrangian

\footnotetext{
${ }^{a}$ Present address: Laboratoire de Physique, Ecole Normale Supérieure de Lyon, Lyon, F-69007 France.

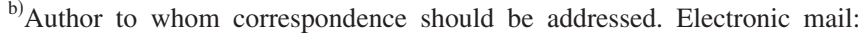
haitao.xu@ds.mpg.de.
}

tracers over inertial time scales. Seminal work was conducted by Snyder and Lumley, ${ }^{9}$ where multiple cameras were used to follow the flow. Only few experiments have been conducted in wind or water tunnels since, with most of them moving the measurement system with the mean flow, ${ }^{10-12}$ or with a measurement volume elongated in the flow direction. ${ }^{13}$ Most recent measurements have been limited to observation times sufficient to measure velocity and acceleration, but not the transport properties of the flow.

In order to allow easier measurements of the Lagrangian properties of turbulent flows, several other types of experimental apparatuses with small mean flow velocities have been devised. The best known example is the von Kármán flow, i.e., the high Reynolds number turbulent water flow between two counter-rotating disks. For the past ten years, it has been the "workhorse" for Lagrangian measurements. ${ }^{2-4,14,15}$ Although, near the center of the apparatus, the mean velocities are much weaker than the fluctuations, the inhomogeneity and anisotropy make the interpretation of the results difficult. In fluids, oscillating grids ${ }^{16,17}$ are able to generate nearly homogeneous and isotropic turbulence with a small mean flow. The achievable Reynolds numbers, however, are small compared to that of turbulent von Kármán flows in laboratory scale devices. Variano et al. ${ }^{18}$ used an array of random jets to create an approximately homogeneous and isotropic turbulent water flow in an open tank, again the Reynolds numbers were quite small. Hwang et al. ${ }^{19}$ used eight speakers at the corners of a cube to drive turbulence in air. Results from two-dimensional particle image velocimetry (PIV) showed that the apparatus generated turbulence with very small mean flow and with a high degree of homogeneity and isotropy at Reynolds numbers comparable to those of oscillating grids. Similar devices utilizing 

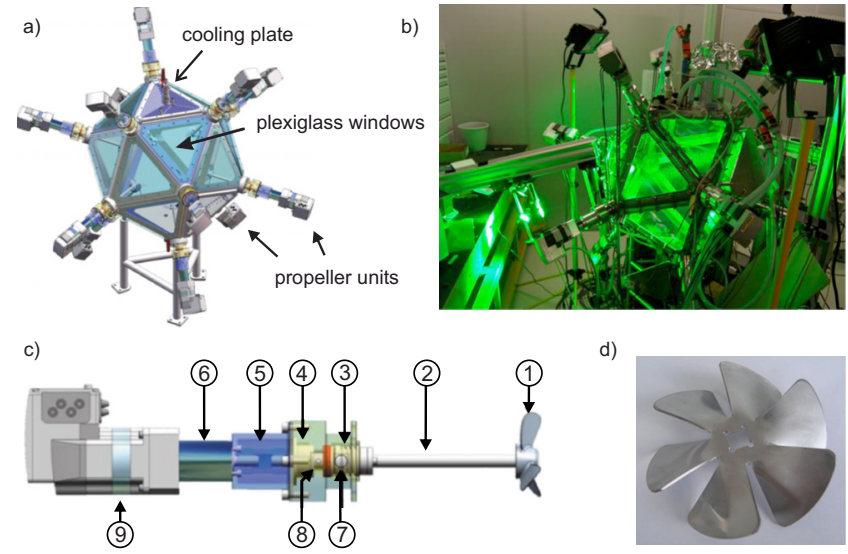

FIG. 1. (Color online) (a) A CAD drawing of the LEM. (b) A photograph of the LEM in operation. An expanded frequency-double Nd:YAG laser beam illuminates the measurement volume. Three high-speed CMOS cameras record the motion of tracer particles from different viewing angles. (c) A CAD drawing of the propeller unit. 1: propeller, 2: exchangeable propeller shaft, 3: rotating seal, 4: bearing, 5: shaft coupling, 6: gearbox, 7: air passage to the bubble trap, 8: ventilation hole for water vapor to escape, 9: brushless dc motor. (d) Stainless steel propeller with a diameter of $10 \mathrm{~cm}$.

multiple jets to drive turbulence in both air and water have been developed later. ${ }^{20-22}$ In a more recent development, 32 spherically arranged speakers are used to generate a turbulent gas flow that has tunable isotropy and homogeneity. ${ }^{23}$

Here we present a novel apparatus, the Lagrangian exploration module (LEM). By comparing Eulerian statistics obtained from three-dimensional Lagrangian particle tracking (LPT) measurements with theoretical relationships derived for homogeneous and isotropic turbulence, we show that the LEM can generate statistically highly homogeneous and isotropic turbulence, as well as turbulence with tunable isotropy and homogeneity.

\section{DESCRIPTION OF THE APPARATUS}

The Max Planck Institute of Dynamics and SelfOrganization in Göttingen designed and constructed the LEM in cooperation with the Laboratoire de Physique of the Ecole Normale Supérieure de Lyon. The apparatus is shaped as a regular icosahedron. Twelve independently controlled propellers, one at each vertex, are used to create turbulent flows with little mean velocity in the central region of the device. By independently controlling each propeller, different forcing schemes can be explored. For isotropic and constant forcing all propellers are rotating with constant rotation frequency; for isotropic and random forcing again all propellers are used, but the frequency and direction of each propeller is randomly changed after random time intervals. When only few propellers are used the flow is anisotropic and for two opposing propellers the von Kármán counter-rotating flow is realized. Here we report data on isotropically and constantly forced flows at Taylor microscale Reynolds number up to $R_{\lambda} \approx 350$. The flows were characterized by LPT. A photograph of the apparatus during LPT measurement is shown in Fig. 1(b). Currently a second device, which has the same icosahedral shape, but has motors installed on 12 of its 20 faces, is being put into operation at the ENS de Lyon.
The apparatus and the experimental setup are described in the following six subsections. First the technical details of the LEM are presented, followed by a detailed description of the LPT system.

\section{A. Basic structure}

The LEM has a stainless steel, skeletal structure in the shape of an icosahedron. A computer-aided design (CAD) drawing is provided in Fig. 1(a). The length of the edges of the icosahedron is $40 \mathrm{~cm}$, which leads to a total volume of 140 1. An O-ring is placed on each face between the icosahedron skeleton and the confining plate. To avoid corrosion the material of all parts were made of plastics or stainless steel. The side plates were either made of Plexiglas [poly(methyl methacrylate)] for optical observation or stainless steel for cooling. The apparatus was mounted on three legs with the middle at a height of $80 \mathrm{~cm}$.

\section{B. Motor and propeller unit}

The design of a propeller unit is shown schematically in Fig. 1(c). Both the propeller and the propeller shaft could be easily exchanged. This allowed maximum flexibility in testing different shaft lengths and propeller designs. The inner part of the shaft was held by a double-row ball bearing. A ceramic seal (Pac Seal, from Flowserve Cooperation, Irving, TX) was installed on each shaft. A threaded hole above the rotating seal on the water side was used for particle insertion or bubble removal (to be described in detail in the next section). Another hole on the dry side of the seal allowed possible water vapor to leave the housing of the propeller unit. The propeller shaft was driven by a brushless dc motor (IFE71, Berger-Lahr, Germany) through a planetary gear box that reduced the motor speed by a ratio of $5: 1$. The gearbox and the propeller shaft were connected by a stainless steel flexible coupler. For the experiments reported here, we used home-made six-blade round stainless steel disks of $10 \mathrm{~cm}$ in diameter, and a helix angle of approximately $60^{\circ}$. A photo of the propeller is shown in Fig. 1(d).

Each of the 12 motors was powered with a constant dc voltage of $36 \mathrm{~V}$ and controlled through a CAN-bus (controller area network). A laptop through a CAN-universal serial bus (USB) adapter (PCAN-USB, Peak Systems GmbH, Germany) controlled via CANopen commands the direction, the rotation frequency, and the angular acceleration of each motor. The CANopen protocol allows up to 127 devices at a data rate of $125 \mathrm{kbits} / \mathrm{s}$, which was more than enough for this purpose. A user defined protocol adjusted the speed and the rotational direction of each motor at specified times. The rotation frequency of the propeller ranged from 1 to 16.67 Hz. As the maximum mechanical power of each motor was $120 \mathrm{~W}$, the upper limit for the turbulent energy dissipation rate was $10 \mathrm{~m}^{2} / \mathrm{s}^{3}$. After filling with water, a small amount of air, as well as dust or lint, were always present inside the apparatus. As bubbles and dust interfere with particle tracking, they needed to be removed before measurements. 
a)

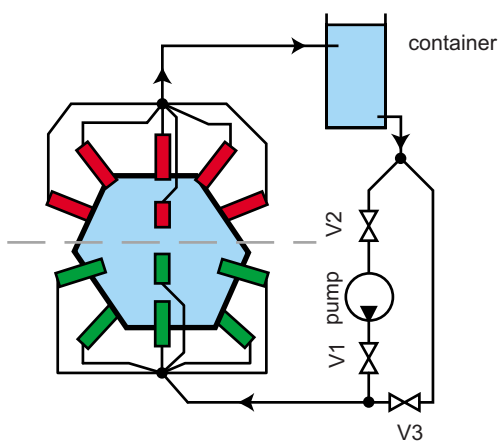

b)

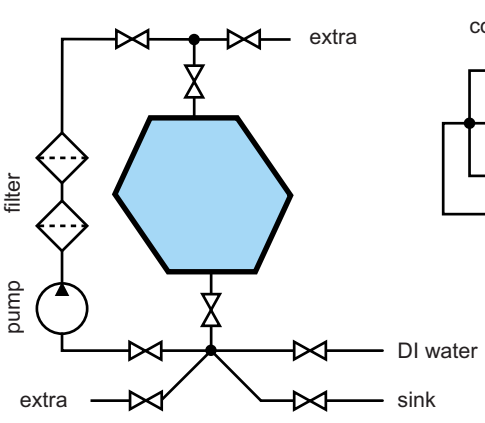

c) cooling

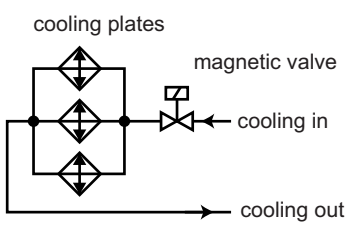

FIG. 2. (Color online) (a) Particle insertion and degassing: When the propellers were rotating, air bubbles accumulated in the low pressure region near the shaft of the six upper propeller units. By pumping water from the upper six propeller units to a gas/water exchange container and from there back to the lower six propeller units, gas bubbles could be removed or tracer particles could be inserted into the LEM. By switching a filter into the circulation circuit the water was filtered to $5 \mu \mathrm{m}$. The three valves (V1, V2, and V3) allowed pressurizing or depressurizing of the LEM. (b) Schematic of the plumbing system for filling, draining, and filteration of the water. (c) Schematic of the temperature control system: A microcontroller regulated the temperature to the set point by controlling the flow of building supplied cooling water with a solenoid valve.

\section{Particle insertion, degassing, and filtration system}

Figure 2 shows schematics of the degassing, particle insertion, and filtration system. Air was removed by pumping fluid from the six upper propeller units [see part 7 in Fig. 1(c)] through a gas/water exchange container and from there back to the lower six propeller units. Degassing was most efficient when at any given time only two opposing propellers were driven in the same direction. This created a vortex, in which air bubbles were trapped. When the propellers were stopped the air bubbles floated upwards and were removed by the circulation into the gas/water exchange container. By randomly switching on and off pairs of propellers air bubbles that adhered to the walls could be efficiently removed. For efficient degassing it was also advantageous to raise the temperature of the water to about $40{ }^{\circ} \mathrm{C}$. Simultaneous with the air removal a secondary water circulation was used for filtration. A pump drew water from an outlet on the bottom plate, pushed it through two filters [the first $100 \mu \mathrm{m}$ and the second $5 \mu \mathrm{m}$ (both from ThermoFisher Scientific)], and then pumped it back to the LEM through the inlet on the top plate. This is shown schematically in Fig. 2(b). Experience showed that $24 \mathrm{~h}$ were sufficient to complete the filtration and degassing procedures. The air removal system could also be used to introduce particle and/or other additives (such as premixed polymer solutions) via the gas/water exchange container.

It was also possible to pressurize or depressurize the working fluid in the apparatus by connecting the gas/water exchange container to a vacuum pump or a high pressure bottle. Evacuation is useful to visualize vortex filaments and to study cavitation in turbulence. ${ }^{24}$ Pressurization is useful to avoid cavitation. In both cases, the pumps had to be bypassed by the valves shown in Fig. 2(a).

\section{Temperature control}

As turbulence transforms all the kinetic energy delivered by the propellers to the flow into heat, it is necessary to temperature control the apparatus in order to avoid a drift in fluid properties. For particle tracking experiments at least four faces of the LEM needed to be covered with poly- acrylamide windows (three for cameras and one for laser illumination), while the others could be covered with cooling plates for temperature control. For the power delivered by the motors three cooling plates were found to be sufficient for maintaining the temperature of the fluid: one on the top, one on the bottom, and one opposing the illuminating laser beam. In principle, it was easy to extend the cooling capacity by adding further cooling plates.

Each cooling plate consisted of a labyrinthian water channel machined into a stainless steel body through which building supplied cooling water was circulated. The cooling power of the plates was adjusted by regulating the flux of the cooling water with a microprocessor (C-Control 2, Conrad, Germany) controlled solenoid valve. Two PT100 resistance temperature detectors were mounted inside the LEM. Their resistances were converted to voltage signals, offsetsubtracted, amplified, and then converted by a 10-bit analogdigital (AD) converter. The microcontroller then compared the temperature averaged over $2 \mathrm{~s}$ with the set point and either opened the solenoid valve or closed it. A sketch of the cooling circuit is shown in Fig. 2(c). The accuracy of the temperature control was $0.4 \mathrm{~K}$ mostly due to internal noise of the $\mathrm{AD}$ converter in the microcontroller. In all experiments presented here the water temperature was at $(18.0 \pm 0.4)^{\circ} \mathrm{C}$.

\section{E. LPT setup}

The LPT system used was similar to that described in Refs. 14 and 15. It consisted of three cameras, a high-power pulsed neodymium-doped yttrium aluminum garnet (Nd:YAG) laser, a master personal computer (PC) for control, and a computer cluster for storing and processing the images. Figure 3 shows a sketch of the wiring diagram for the LPT system. Three Phantom V7.2 high-speed complementary metal oxide semiconductor (CMOS) cameras (Vision Research, Wayne, NJ) recorded 10-bit grayscale images at frame rates of up to 11000 frames per second at a resolution of $512 \times 512$ pixels. Only the upper 8 bit of each image were read out via gigabit-ethernet into the computer cluster. The synchronization of the image capture from the three cameras were achieved by illuminating the measure- 


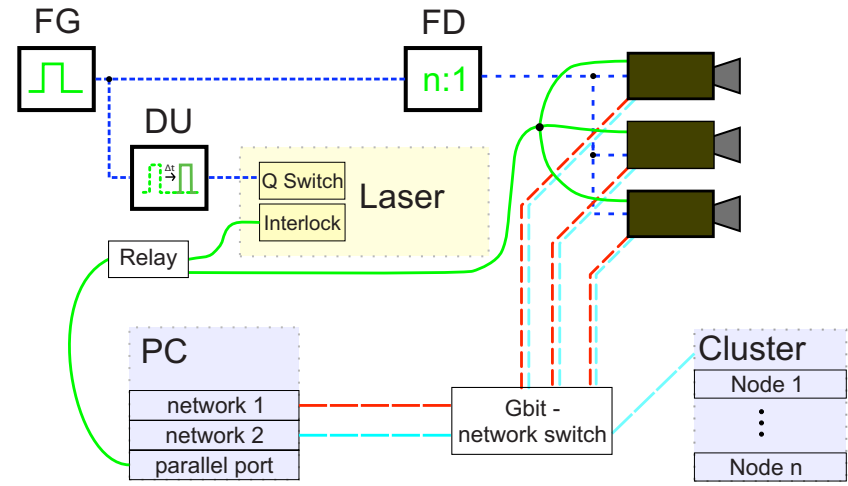

FIG. 3. (Color online) Schematic wiring of the LPT system: for details see text. FG generates a pulse train. FD is the frequency divider and DU is the delay unit. A master PC controlled the image capture and laser shutter through the parallel port. The images from the three cameras were downloaded via ethernet to the computer cluster for analysis.

ment volume with a pulsed laser, which in turn was triggered by a waveform generator (Agilent Technologies, Santa Clara, CA). The laser could only run at repetition rates higher than $20 \mathrm{kHz}$, and thus was faster than the camera frame rate used. Synchronization was achieved by triggering the laser at a frequency that was an integer times the camera frame rate and by triggering the cameras with the appropriate frequency divided signal. It was important that the laser trigger was delayed by a few microseconds in order to compensate for the internal response time of the cameras.

For each experiment, the frame rate of the cameras was selected based on the Kolmogorov time scale. Initially it was estimated and later extrapolated based on existing measurements. The frame rate was selected one or two orders of magnitude faster than the Kolmogorov time scale ${ }^{3,25}$ since Lagrangian properties of turbulence are highly intermittent, ${ }^{6}$ especially the acceleration. ${ }^{3}$ Details on the frame rates in units of frames per Kolmogorov timescale are listed in Table I.

Sieved hollow glass spheres (MO-SCI cooperation), with diameters of 60-70 $\mu \mathrm{m}$ were used as tracer particles. These hollow spheres have a wide range of density, ranging between 0.8 and $2 \mathrm{~g} / \mathrm{cm}^{3}$, but the majority is within 1 and $1.2 \mathrm{~g} / \mathrm{cm}^{3}$, approximately the same as the water density. The inertial effect and the Stokes numbers, $S t$, of these particles are discussed later.

The laser was a frequency-doubled, pulsed Nd:YAG laser (DDC Technologies, Oceanside, NY) with a wavelength of $532 \mathrm{~nm}$ and pulse width of $200 \mathrm{~ns}$. The output power was approximately $30 \mathrm{~W}$ over a repetition rate range between 20 and $100 \mathrm{kHz}$. The laser beam was collimated and expanded to $15 \mathrm{~cm}$ in diameter. The whole system was aligned so that the beam entered the LEM perpendicular to the window surface and passed through the center of the apparatus. The scattering light can be seen in Fig. 1(b), a photograph taken while the LEM was in operation. The measurement volume was approximately $15 \times 10 \times 10 \mathrm{~cm}^{3}$ in size, as defined by the overlapping region among the observation volumes from all three cameras. The spatial resolution of the imaging system was $0.2 \mathrm{~mm}$ per pixel. The optical system was calibrated with an optical mask as described in Ref. 26.

The LPT system was controlled by a master PC (master), which set the parameters, such as the frame rate, frame format, exposure time, etc., as well as the operation sequence of the different components. In addition, five to ten nodes (slaves) in a computer cluster (Dell PowerVault) were used for image analysis. For each recording cycle the master first armed the laser by opening the shutter. After a few seconds, when the laser had stabilized, the master sent a "recording" command to the cameras. After acquiring images for the given recording time (typically less than a second due to the size of the onboard memory of the cameras), the master stopped the camera recording and closed the laser shutter. This "synchronous stop" allowed the master to instruct a slave to download the acquired images from the three cameras, counting backward, ensuring synchronization of the images. After downloading (typically 3-5 $\mathrm{min}$ ) the slave issued an "end of downloading" (EoD) signal to the master, and started calculating the particle trajectories using the algorithm described in Ref. 26. After having received the EoD from the node the master started the next recording cycle and selected the next slave from a list for download. The process continued until all the slaves on the list had been called upon. The number of nodes was selected so that the first slave was finished with calculating trajectories by the time

TABLE I. Parameters of the experiments. $f$ is the propeller frequency. $u^{\prime} \equiv\left(u_{x}^{\prime}+u_{y}^{\prime}+u_{z}^{\prime}\right) / 3$ is the fluctuation velocity, averaged over the entire measurement volume. $\varepsilon$ is the turbulence energy dissipation rate per unit mass, measured from the inertial range scaling of the Eulerian velocity structure functions. $R_{\lambda}$ is the Taylor microscale Reynolds number, calculated from Eq. (4). $L \equiv u^{\prime 3} / \varepsilon$ is the integral scale. $\eta$ and $\tau_{\eta}$ are the Kolmogorov length and time scales of the flow, respectively. $S t \equiv \tau_{p} / \tau_{\eta}$ is the Stokes number of tracer particles, where $\tau_{p} \equiv\left(2 \rho_{p}+\rho_{f}\right) d_{p}^{2} /\left(36 \mu_{f}\right)$ is the viscous relaxation time of the particles and $\rho_{p}=1.1 \mathrm{~g} / \mathrm{cm}^{3}$ and $d_{p}=65 \mu \mathrm{m}$ are used. $f_{\text {cam }}$ is the camera frame rate in units of frames per $\tau_{\eta} .|u|$ is the average of the magnitude of the mean flow in the measurement volume.

\begin{tabular}{lccccccccc}
$\begin{array}{c}f \\
(\mathrm{~Hz})\end{array}$ & $\begin{array}{c}u^{\prime} \\
(\mathrm{cm} / \mathrm{s})\end{array}$ & $\begin{array}{c}\varepsilon \\
\left(\mathrm{m}^{2} / \mathrm{s}^{3}\right)\end{array}$ & $R_{\lambda}$ & $\begin{array}{c}L \\
(\mathrm{~cm})\end{array}$ & $\begin{array}{c}\eta \\
(\mu \mathrm{m})\end{array}$ & $\begin{array}{c}\tau_{\eta} \\
(\mathrm{ms})\end{array}$ & $S t$ & $\begin{array}{c}f_{\text {cam }} \\
\left(\mathrm{frame} / \tau_{\eta}\right)\end{array}$ & $\begin{array}{c}|u| \\
(\mathrm{cm} / \mathrm{s})\end{array}$ \\
\hline 1 & 1.5 & $(3.24 \pm 0.15) \times 10^{-5}$ & $150 \pm 10$ & $10 \pm 1$ & $420 \pm 5$ & $175 \pm 4$ & $2.1 \times 10^{-3}$ & 175 & 0.1 \\
1.67 & 2.7 & $(2.14 \pm 0.03) \times 10^{-4}$ & $195 \pm 10$ & $9.4 \pm 0.6$ & $261 \pm 1$ & $68 \pm 0.5$ & $5.5 \times 10^{-3}$ & 68 & 0.1 \\
2.25 & 3.6 & $(6.33 \pm 0.15) \times 10^{-4}$ & $200 \pm 8$ & $7.3 \pm 0.5$ & $200 \pm 1$ & $40 \pm 0.5$ & $9.4 \times 10^{-3}$ & 40 & 0.3 \\
5 & 6.2 & $(5.2 \pm 0.8) \times 10^{-3}$ & $210 \pm 45$ & $5 \pm 1.6$ & $118 \pm 4$ & $14 \pm 1$ & $2.7 \times 10^{-2}$ & 35 & 0.5 \\
7.5 & 9.7 & $(1.4 \pm 0.2) \times 10^{-2}$ & $300 \pm 60$ & $6 \pm 2$ & $91 \pm 4$ & $8.4 \pm 0.6$ & $4.5 \times 10^{-2}$ & 42 & 1.2 \\
9.33 & 12.6 & $(3.6 \pm 0.5) \times 10^{-2}$ & $330 \pm 70$ & $6 \pm 2$ & $73 \pm 3$ & $5.3 \pm 0.3$ & $7.1 \times 10^{-2}$ & 26.5 & 1.1 \\
\hline \hline
\end{tabular}




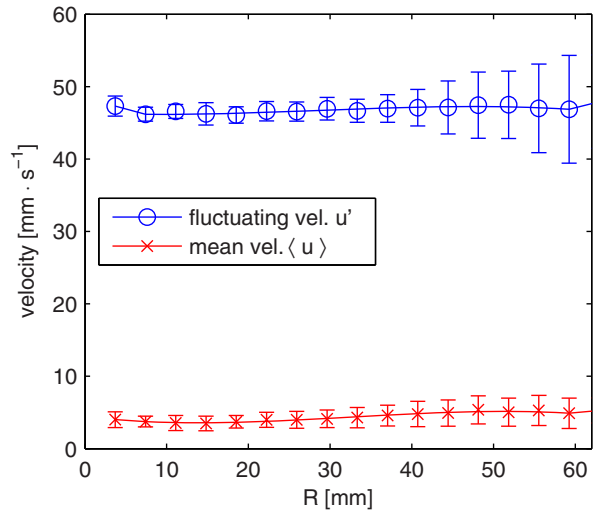

FIG. 4. (Color online) The magnitudes of the mean $|u|$ and the fluctuating velocities $u^{\prime}$ averaged in spherical shells around the center of the measurement volume, vs the radius measured from he center. The error bars indicate the standard deviation within each shell. $R_{\lambda}=195$.

the master selected it for the next download. After the experimental run, which consisted of many recording cycles, all the trajectories were processed with the algorithm in Ref. 27 to connect interrupted segments resulted from fluctuation in illumination, particle shading, and pixel blinking.

\section{RESULTS}

In this section, we report the quantitative measurements in the LEM at different propeller speeds. The particle velocities were calculated with a smoothing and differentiating kernel. $^{28}$ Eulerian statistics were measured by simultaneously tracking several hundreds of particles.

The energy dissipation rate, $\varepsilon$, was determined from the well-known scalings of the velocity structure functions in the inertial range (see, e.g., Ref. 29)

$$
\begin{aligned}
& \varepsilon=\frac{1}{r}\left[\frac{D_{L L}(r)}{C_{2}}\right]^{3 / 2}, \\
& \varepsilon=\frac{1}{r}\left[\frac{3 D_{N N}(r)}{4 C_{2}}\right]^{3 / 2},
\end{aligned}
$$

and

$$
\varepsilon=-\frac{5 D_{L L L}(r)}{4 r},
$$

where $D_{L L}(r)$ and $D_{N N}(r)$ are the second-order longitudinal and transverse structure functions, $D_{L L L}(r)$ is the third-order longitudinal structure function, and $C_{2}$ is an universal constant. The currently accepted value for $C_{2}=2.1$ was used. ${ }^{30}$ Although these scaling relations are strictly applicable only to statistically homogeneous and isotropic turbulence, experience $^{31}$ shows that they work well even in other situation. As we will find, the LEM can produce statistically homogeneous and isotropic turbulence. Therefore, in this case the application of the scaling relations if fully justified. All three methods give similar values and here the average $\varepsilon$ is reported.

The Taylor microscale Reynolds number of the flow, $R_{\lambda}$, was also calculated based on the assumption of statistically homogeneous and isotropic turbulence (see, e.g., Ref. 29)

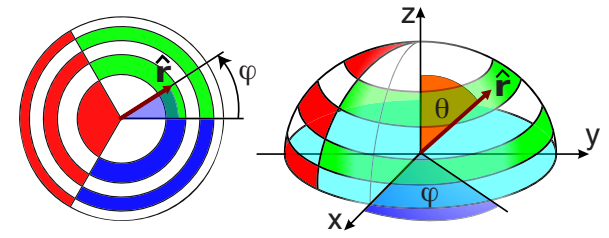

FIG. 5. (Color online) Grouping of the separation vectors on a unit hemisphere with $3 \times 5$ bins. Each bin covers the same solid angle.

$$
R_{\lambda}=\frac{u^{\prime} \lambda}{\nu}=\frac{\sqrt{15} \tau_{\eta} u^{\prime 2}}{\nu}=\sqrt{\frac{15 u^{\prime 4}}{\nu \varepsilon}},
$$

where $u^{\prime}$ is the turbulence fluctuating velocity and $\tau_{\eta}$ denotes the Kolmogorov time scale.

The integral length scale, $L$, which characterizes the largest eddies of the turbulent flow, is estimated from the relationship

$$
L=C_{L} u^{\prime 3} / \varepsilon,
$$

where $u^{\prime}$ is the turbulence fluctuating velocity and $C_{L}$ is a dimensionless constant. For $R_{\lambda} \leq 400$, a collection of available experimental and numerical data suggests that $0.5 \lesssim C_{L} \lesssim 1.5,{ }^{32,33}$ so here $C_{L}=1$ in Eq. (5) was used for the estimation of the parameters reported in Table I. Please note that $L$ can also be estimated from the decay of the longitudinal and transverse velocity correlations. This yielded for all $R_{\lambda}$ an integral scale of $\approx 4.5 \mathrm{~cm}$, in good agreement with the values given in Table I. Discrepancies could be attributed to the unknown Reynolds number dependence of $C_{L}$. In the following we present mostly results for $R_{\lambda}=195$; other Reynolds numbers gave similar results.

\section{A. Statistical homogeneity}

The statistical homogeneity of the velocity field was quantified by dividing the observation volume into spherical, "onion" shells of radius $R$ around the center of the measurement volume and by calculating the fluctuating and the mean velocities in each shell. Figure 4 shows the mean and the fluctuating velocities averaged over the spherical shells versus $R$. The error bars in Fig. 4 indicate the standard deviation within each shell. Both the fluctuating and the mean velocities were observed to be nearly constant up to $R \approx 4 \mathrm{~cm}$. Thus, the flow was observed to be statistically homogeneous up to the integral length scale $L$ (see Table I) of the turbulence. The increasing uncertainties with increasing $R$ was due to worsening counting statistics: The intensity of the laser illumination decreased at the outer shells and less particle trajectories were observed. Thus, it is likely that the homogeneous region was even larger. Figure 4 also indicates that the magnitude of the mean velocity is much smaller than the fluctuating velocity throughout the measurement volume.

\section{B. Statistical isotropy}

The statistical isotropy of the flow was quantified by the second order longitudinal and transverse velocity structure functions $D_{L L}(\mathbf{r})$ and $D_{N N}(\mathbf{r})$ conditioned on the direction of the separation vector $\mathbf{r}$. The separation vectors $\mathbf{r}$ were grouped into bins that each occupied the same solid angle. Figure 5 shows schematically this grouping. Then the struc- 


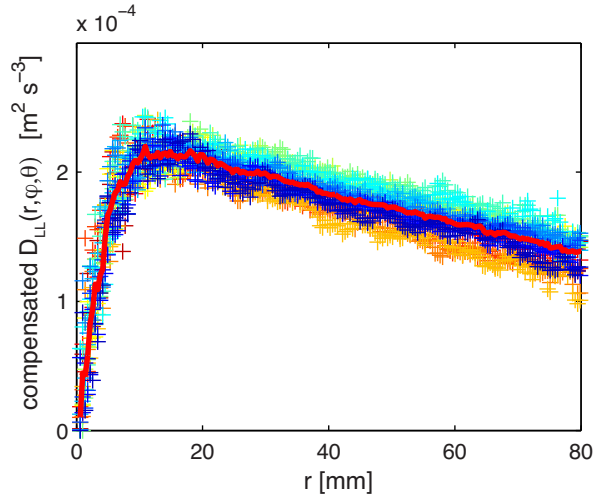

FIG. 6. (Color online) Independence of the longitudinal velocity structure function, $D_{L L}(\mathbf{r})$ on the direction of the separation vector $\mathbf{r}$. The structure functions were compensated as in Eq. (1) and the maximum value gives the energy dissipation rate $\varepsilon$. The symbols show the data for 15 bins of $\mathbf{r}$ in different directions, but spanning the same solid angle. The line is the average. $R_{\lambda}=195$

ture functions $D_{L L}(\mathbf{r})$ and $D_{N N}(\mathbf{r})$ for $\mathbf{r}$ were calculated in each bin. As shown in Fig. 6, the longitudinal structure function $D_{L L}(\mathbf{r})$ calculated from all $15(\varphi, \theta)$ bins collapsed well, i.e., it is indeed independent of the direction of $\mathbf{r}$ and thus isotropic. The same was true for $D_{N N}(\mathbf{r})$.

In statistically homogeneous and isotropic turbulence, the structure functions $D_{L L}(r)$ and $D_{N N}(r)$ are related by (see, e.g., Ref. 29)

$$
D_{N N}(r)=D_{L L}(r)+\frac{r}{2}\left[\frac{\partial}{\partial r} D_{L L}(r)\right]
$$

or equivalently

$$
D_{L L}(r)=\frac{2}{r^{2}} \int_{0}^{r} x D_{N N}(x) d x .
$$

If either $D_{L L}(r)$ or $D_{N N}(r)$ is known, the above equations can be used to calculate the other. By comparing the measured and calculated values, the degree of isotropy can be estimated. Figure 7 compares the measured structure functions (superscript ${ }^{\mathrm{m}}$ ) with the calculated ones (superscript ${ }^{\text {th}}$ ). For both $D_{L L}(r)$ and $D_{N N}(r)$, the relative deviations were below $10 \%$ for separations smaller than $8 \mathrm{~cm}$. The slightly larger relative deviations in $D_{N N}(r)$ can be attributed to the numerical differentiation when calculating $D_{N N}^{\text {th }}$ from Eq. (6).

The statistical isotropy can also be quantified by a directional analysis of the coarse-grained velocity gradients at the integral scale. A method similar to that in Ref. 34 was used over the whole measurement volume. The coarse-grained velocity gradients can be decomposed into the "large-scale" vorticity and the rate-of-strain tensors. Figures 8(a) and 8(b) show the distribution of the direction of the coarse-grained vorticity vectors $\omega$ and the eigenvectors $\mathbf{e}_{1}$ corresponding to the largest eigenvalues of the coarse-grained rate-of-strain tensors, as measured by the cosines of the angles between the three axes of the laboratory coordinates and these two vectors. If the flow is isotropic, both vectors $\omega$ and $\mathbf{e}_{1}$ should orient randomly in space and the probability density functions (PDFs) of the angular cosines should be flat, i.e., a uniform distribution. Figures $8(\mathrm{a})$ and $8(\mathrm{~b})$ show that this was the case for the turbulent flow in the LEM. As a com-

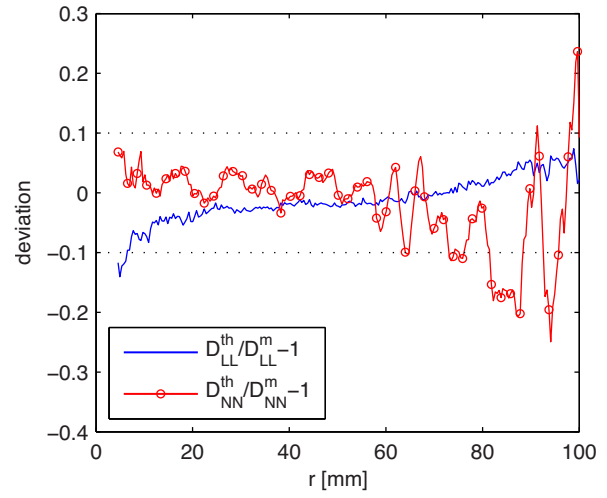

FIG. 7. (Color online) Relative deviations of the measured longitudinal and transverse velocity structure functions from the theoretical values that were calculated under the assumption of statistical homogeneity and isotropy. The horizontal dotted lines mark the $\pm 10 \%$ deviations. $R_{\lambda}=195$.

parison, the same PDFs were analyzed for a von Kármán flow using the experimental data from Refs. 14 and 15. The results are shown in Figs. 8(c) and 8(d), in which the coordinate system is chosen such that the axis of rotation of the propellers is the $z$-axis. The axial symmetry of the forcing can be seen in these PDFs. In comparison, the LEM successfully produced highly isotropic flows.

\section{Reynolds number effects}

The turbulence properties were also measured at different driving speeds of the propellers, ranging from 1 to 9.33 $\mathrm{Hz}$, as shown in Table I. At constant observation volume the maximum achievable Reynolds number was limited by the laser illumination and not by the power of the motors. As the Reynolds number increases, the Kolmogorov time scale $\tau_{\eta}$ decreases. If one assumes that the integral length scale is constant, then $\tau_{\eta}$ decreases as $R_{\lambda}^{-3 / 2}$. Thus reliable measurement of the fastest motions of the flow requires the camera frame rate to increase with $R_{\lambda}$. On the other hand, the laser delivered constant average power and not a constant energy per pulse. In other words, the available number of photons per frame decreased with increasing frame rates and thus limited the maximal useable frame rate. At a motor frequency of $9.33 \mathrm{~Hz}$ and a camera frame rate of 5000 frames per second the image quality was already borderline.

For all Reynolds numbers, the tracer particles were smaller than the Kolmogorov scale of the turbulent flow. To characterize the effect of particle inertia, Table I lists the particle Stokes number, defined as the ratio of the particle relaxation time $\tau_{p} \equiv\left[\left(2 \rho_{p}+\rho_{f}\right) d_{p}^{2}\right] / 36 \mu_{f}$ to the Kolmogorov time scale $\tau_{\eta}$. The Stokes numbers are much smaller than one and hence the particles may be considered tracers.

At all the speeds, the flow was observed to be nearly statistically homogeneous and isotropic and the mean flow was small compared to the fluctuations. Figure 9 shows the mean and rms fluctuation of the three velocity components and the ratio of the mean to the fluctuation. In the range of Reynolds number explored that ratio was observed to be less than $15 \%$ and no systematic trend was observed.

One interesting observation is that the integral scale of the flow, as calculated from Eq. (5), decreased with propeller speed (or $\left.R_{\lambda}\right)$ at low speed $\left(R_{\lambda} \leq 200\right)$ before reaching a con- 
alignment between global vorticity and lab coordinates
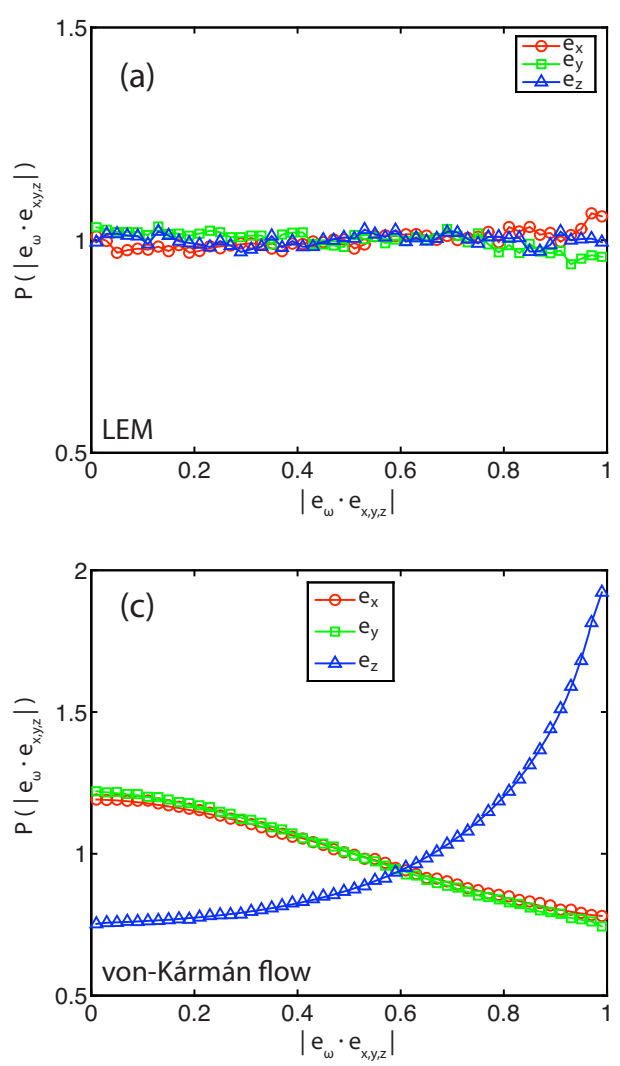

alignment between global strain and lab coordinates
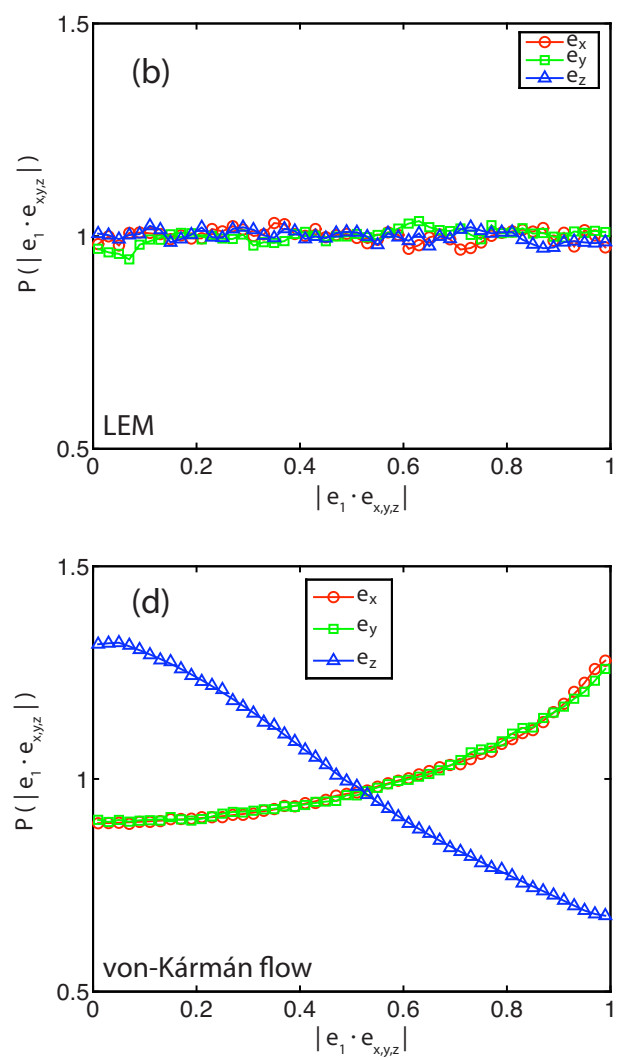

FIG. 8. (Color online) [(a) and (c)] PDFs of the alignment of the coarse-grained large scale vorticity vector $\omega(t)$ with the axes of laboratory coordinates. [(b) and (d)] PDFs of the alignment of the eigenvector $\mathbf{e}_{1}$, corresponding to the largest eigenvalue of the coarse-grained large scale rate of strain tensor. (a) and (b) are from the LEM at $R_{\lambda}=195$. (c) and (d) are from the von Kármán device at $R_{\lambda}=690$ (Refs. 14 and 15).

stant of approximately $6 \mathrm{~cm}$. This is slightly different from the von Kármán flow reported in Ref. 25, where the integral scale was approximately independent of the propeller speed. It is possible that the particular shape of the propellers affects the turbulence at low Reynolds numbers through the mean flow field. This we also had observed in a von Kármán flow driven with a different propeller design. ${ }^{35}$ It is also possible that the dimensionless constant $C_{L}$ in Eq. (5) depends on Reynolds number and flow geometry.

\section{SUMMARY AND OUTLOOK}

We constructed an apparatus, the LEM, that generated laboratory flows by 12 independently controlled propellers arranged symmetrically in three dimension (3D). Results from 3D LPT measurements demonstrated that at the center of the apparatus a region comparable in size to that of the integral length scale of the turbulence was statistically homogeneous and isotropic. Moreover, the mean flow was at least one order of magnitude smaller than the turbulent fluctuating velocities. These features make the LEM an attractive device for studying the Lagrangian properties of turbulence. The large number of flat windows on the LEM can be used to simultaneously carry out several measurements using different techniques, for example, Eulerian measurements using PIV and Lagrangian measurements using particle tracking.

Figure 10 shows that the turbulent fluctuating velocity in the LEM varied linearly with propeller speed, as in many other laboratory disk/propeller driven flows (see, e.g.,
Ref. 25). If the linear fit in Fig. 10 is extrapolated to the maximum motor speed and assuming that the integral scale remained at $L \approx 6 \mathrm{~cm}$, then the highest Reynolds number would be $R_{\lambda} \approx 450$. In that case, the maximum turbulent energy dissipation rate per unit mass is $\varepsilon \approx u^{\prime 3} / L$ $\approx 0.18 \mathrm{~W} / \mathrm{kg}$, which means that the propellers very inefficiently converted mechanical power into turbulent kinetic energy. By changing the design of the propellers, e.g., by using propellers with larger blades it should be possible to

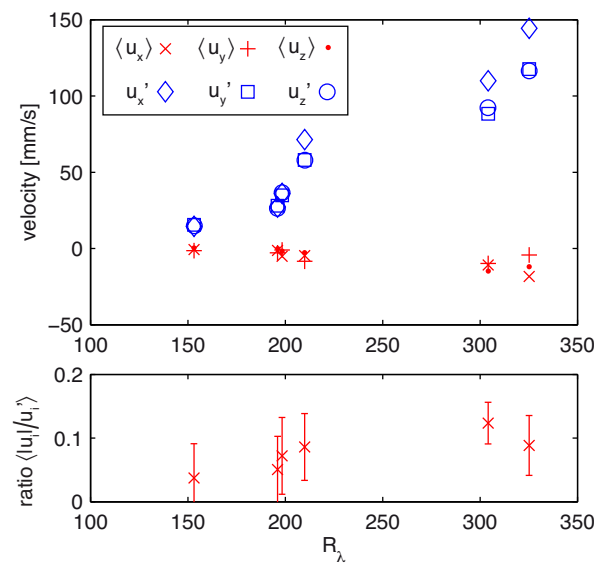

FIG. 9. (Color online) Top: components of the mean and fluctuating velocities, averaged over the entire measurement volume, at different Taylor scale Reynolds numbers. Bottom: ratio of the mean velocity to the fluctuating velocity, $\left[\left(\left|\left\langle u_{x}\right\rangle\right| / u_{x}^{\prime}\right)+\left(\left|\left\langle u_{y}\right\rangle\right| / u_{y}^{\prime}\right)+\left(\left|\left\langle u_{z}\right\rangle\right| / u_{z}^{\prime}\right)\right] / 3$. The error bars in the bottom plot indicate the standard deviation within the three components. 


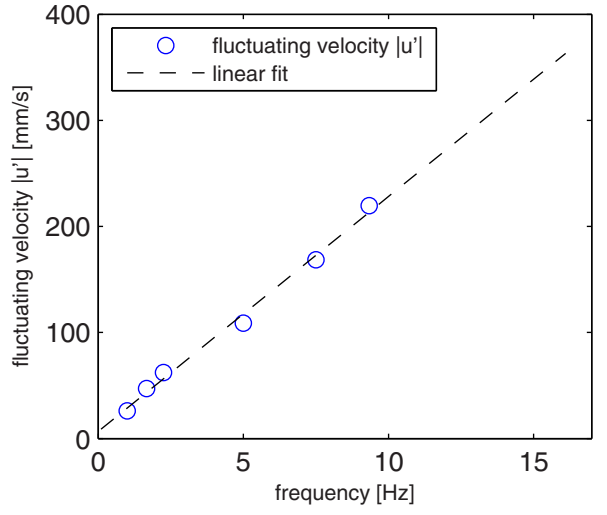

FIG. 10. (Color online) The change in fluctuating velocity with motor frequency.

increase the turbulence level of the flow. Theoretically, a Reynolds number of $R_{\lambda} \approx 900$ could be achieved in the LEM, if it is possible to convert all the mechanical power of the motors into kinetic energy of the flow while keeping the integral length scale at $L \approx 6 \mathrm{~cm}$. This would be comparable with active grids driven turbulence in a moderate size wind tunnel. ${ }^{7}$ This optimistic estimate remains to be examined in the future.

Here we reported only results from driving the propellers at constant speeds and in one rotating direction. More driving patterns were tested, such as randomly modulating the speeds of individual propellers while keeping the energy injection rate constant. ${ }^{36}$ In addition, the LEM allows to gradually change the flow from anisotropic forcing, such as using only a pair of propellers, to the more isotropic forcing as done in an air flow in Ref. 23. It is clear that many more tests and diagnostics must be performed in order to fully quantify the achievable degree of resemblance of such a laboratory flow with ideal homogenous isotropic turbulence. Flows forced in confined geometries are indeed bound to inject energy at many scales, possibly mixing several kinds of dynamics in a way different from the statistical equilibrium assumed theoretically or achieved in direct numerical simulations using volume forcing in a 3D-periodic space. These issues are currently under investigation.

\section{ACKNOWLEDGMENTS}

We are grateful to the machine shop at MPIDS, especially U. Schminke and J. Hesse; and P. Metz and D. Le Tourneau at ENS-Lyon for technical assistances during the construction of the apparatus. This work was supported by the Max Planck Society and by French ANR BLAN 071 192604. We also acknowledge PROCOPE support that initiated this project. This work is based primarily on the Diploma thesis "The Lagrangian Exploration Module" by Robert Zimmermann.

${ }^{1}$ A. N. Kolmogorov, Dokl. Akad. Nauk SSSR 30, 301 (1941).

${ }^{2}$ G. A. Voth, K. Satyanarayan, and E. Bodenschatz, Phys. Fluids 10, 2268 (1998).

${ }^{3}$ A. La Porta, G. A. Voth, A. M. Crawford, J. Alexander, and E. Bodenschatz, Nature (London) 409, 1017 (2001).

${ }^{4}$ N. Mordant, P. Metz, O. Michel, and J.-F. Pinton, Phys. Rev. Lett. 87, 214501 (2001)

${ }^{5}$ G. Falkovich, K. Gawedzki, and M. Vergassola, Rev. Mod. Phys. 73, 913 (2001)

${ }^{6}$ F. Toschi and E. Bodenschatz, Annu. Rev. Fluid Mech. 41, 375 (2009).

${ }^{7}$ L. Mydlarski and Z. Warhaft, J. Fluid Mech. 320, 331 (1996).

${ }^{8}$ A. K. Kuczaj, B. J. Geurts, D. Lohse, and W. van de Water, Comput. Fluids 37, 816 (2008).

${ }^{9}$ W. H. Snyder and J. L. Lumley, J. Fluid Mech. 48, 41 (1971).

${ }^{10}$ Y. Sato and K. Yamamoto, J. Fluid Mech. 175, 183 (1987).

${ }^{11}$ S. Ayyalasomayajula, A. Gylfason, L. R. Collins, E. Bodenschatz, and Z. Warhaft, Phys. Rev. Lett. 97, 144507 (2006).

${ }^{12}$ M. Virant and T. Dracos, Meas. Sci. Technol. 8, 1539 (1997).

${ }^{13}$ N. M. Qureshi, M. Bourgoin, C. Baudet, A. Cartellier, and Y. Gagne, Phys. Rev. Lett. 99, 184502 (2007).

${ }^{14}$ M. Bourgoin, N. T. Ouellette, H. Xu, J. Berg, and E. Bodenschatz, Science 311, 835 (2006).

${ }^{15}$ H. Xu, N. T. Ouellette, and E. Bodenschatz, New J. Phys. 10, 013012 (2008).

${ }^{16}$ I. P. D. De Silva and H. J. S. Fernando, Phys. Fluids 6, 2455 (1994).

${ }^{17}$ S. Ott and J. Mann, J. Fluid Mech. 422, 207 (2000).

${ }^{18}$ E. A. Variano, E. Bodenschatz, and E. A. Cowen, Exp. Fluids 37, 613 (2004).

${ }^{19}$ W. Hwang and J. K. Eaton, Exp. Fluids 36, 444 (2004).

${ }^{20}$ D. R. Webster, A. Brathwaite, and J. Yen, Limnol. Oceanogr. Methods 2, 1 (2004)

${ }^{21}$ E. A. Variano and E. A. Cowen, J. Fluid Mech. 604, 1 (2008).

${ }^{22}$ C. Goepfert, J.-L. Marie, D. Chareyron, and M. Lance, Exp. Fluids 48, 809 (2010).

${ }^{23}$ K. Chang, G. Bewley, and E. Bodenschatz, "Manipulating the isotropy of turbulence," J. Fluid Mech. (submitted).

${ }^{24}$ A. La Porta, G. A. Voth, F. Moisy, and E. Bodenschatz, Phys. Fluids 12, 1485 (2000)

${ }^{25}$ G. A. Voth, A. La Porta, A. M. Crawford, J. Alexander, and E. Bodenschatz, J. Fluid Mech. 469, 121 (2002).

${ }^{26}$ N. T. Ouellette, H. Xu, and E. Bodenschatz, Exp. Fluids 40, 301 (2006).

${ }^{27}$ H. Xu, Meas. Sci. Technol. 19, 075105 (2008).

${ }^{28}$ N. Mordant, A. M. Crawford, and E. Bodenschatz, Physica D 193, 245 (2004).

${ }^{29}$ S. B. Pope, Turbulent Flows (Cambridge University Press, Cambridge, England, 2000).

${ }^{30}$ K. R. Sreenivasan, Phys. Fluids 7, 2778 (1995).

${ }^{31}$ M. A. Taylor, S. Kurien, and G. L. Eyink, Phys. Rev. E 68, 026310 (2003).

${ }^{32}$ K. R. Sreenivasan, Phys. Fluids 10, 528 (1998).

${ }^{33}$ B. R. Pearson, P.-Å. Krogstad, and W. van de Water, Phys. Fluids 14, 1288 (2002).

${ }^{34}$ M. Chertkov, A. Pumir, and B. I. Shraiman, Phys. Fluids 11, 2394 (1999).

${ }^{35} \mathrm{H}$. Xu and E. Bodenschatz (unpublished).

${ }^{36}$ R. Zimmermann, “The Lagrangian Exploration Module," Diploma thesis, University of Göttingen, 2008. 